\title{
Idiothetic Verticality Estimation through Head Stabilization Strategy
}

\author{
Ildar Farkhatdinov, Hannah Michalska, Alain Berthoz, and Vincent Hayward
}

\begin{abstract}
The knowledge of the gravitational vertical is fundamental for the autonomous control of humanoids and other freemoving robotic systems such as rovers and drones. This article deals with the hypothesis that the so-called 'head stabilization strategy' observed in humans and animals facilitates the estimation of the true vertical from inertial sensing only. This problem is difficult because inertial measurements respond to a combination of gravity and fictitious forces that are hard to disentangle. From simulations and experiments, we found that the angular stabilization of a platform bearing inertial sensors enables the application of the separation principle. This principle, which permits one to design estimators and controllers independently from each other, typically applies to linear systems, but rarely to nonlinear systems. We found empirically that, given inertial measurements, the angular regulation of a platform results in a system that is stable and robust and which provides true vertical estimates as a byproduct of the feedback. We conclude that angularly stabilized inertial measurement platforms could liberate robots from ground-based measurements for postural control, locomotion, and other functions, leading to a true idiothetic sensing modality, that is, not based on any external reference but the gravity field.
\end{abstract}

Index Terms-Biologically-Inspired Robots; Biomimetics; Sensor-based Control

\section{INTRODUCTION}

For humans, as for other living creatures, it is substantial to know the spatial orientation of our body with respect to the external world. Most of our sensory systems can contribute to this task. Interestingly, visual, auditory, tactile, proprioceptive, or olfactory sensory inputs can be easily put out of action, but the vestibular inputs are always available, even in the absence of gravity [1]. In artificial systems, robots in particular, inertial measurement units (IMUs) often play the role of a 'robotic vestibular system'. These IMUs sense the movements of the robot and provide its control system with data that can be further processed to yield estimates of the robot location and displacement in space. The operating principle of biological motion sensors and of engineering inertial sensors is based on the same laws of mechanics. Thus, we believe that strategies observed in biological systems, such as head stabilization, can also benefit the design of robotic systems.

In all fast moving animals, including fishes, reptiles, amphibians, birds, mammals, in brief, in all vertebrates, the vestibular sensors are located in the head and each comprises two types of organs: two otolith organs and three semicircular canals. The otolith organs, which are responsible for gravitational and acceleration sensing, comprise the utricle and the saccule. They respond to the acceleration of the head in three dimensions and to static tilt relative to vertical planes. When the head is upright, the saccule is vertical and it responds to linear accelerations in the sagittal plane, specifically up and down movements. The utricle is horizontally oriented and responds to accelerations in the interaural transverse (horizontal) plane (anterio-posterior and medio-lateral accelerations). Otoliths, like all accelerometers, are sensitive to the gravitational acceleration vector, and provide us with a sense of absolute verticality [2]. The knowledge of gravitational verticality is essential for balancing and posture control, but more generally, enables the specification of 'up' and 'down' for spatial orientation.

Head stabilization behavior, which is universally observed in humans and animals, is directly related to the functions of the vestibular system alluded earlier [3]. In humans, experimental studies have shown that the head is stabilized during the performance of different locomoting, balancing, and other postural tasks $[4,5]$. The plane of stabilization was determined by the task, could vary, and was controlled by the gaze. The head remained stable relative to the vertical during locomotion. Head angular stabilization close to the vertical orientation was essential for effective postural control during the execution of complex, dynamic tasks. In some cases head stabilization could be related to the skill of a person, such as a dancer or an acrobat. Often too, head stabilization was the result of vestibular-ocular interactions [6]. However, in all of these cases, vestibular information was important for the head-neck control system, and sometimes it was the only source of information available [7]. Additional behavioral studies showed that the orientation of the head anticipates turning during locomotion relative to the walking direction [8]. This observations suggests that head orientation and gaze stabilization are important functions for motion planning during locomotion and that both visual and vestibular cues are processed by the neural system for the head to be stabilized in orientational space.

In robotic systems, inertial sensors could play a role similar to that of the vestibular system in humans and animals. However, most robots have an IMU, or, as we may call it, an artificial vestibular system in the main body regions: hip, torso, trunk, or pelvis. Some robot designers do locate an IMU in the head $[9,10,11]$. The rational for this choice, however, is often unrelated to biological vestibular functions. To our knowledge only very few humanoid robots have inertial sensors in the head for posture control during locomotion and balancing. One example was found in a humanoid robot with two IMUs: one in the head and another one in the torso [12]. In another example, a robot head stabilization control based on orientation was 
demonstrated [13]. However, the performance of such systems was deemed insufficient owing to the latency of the sensory feedback. One additional exception was a proposal for a feedback learning algorithm based on artificial neural networks employed to stabilize head orientation independently from the trunk motions [14]. In other studies it was suggested that head and trunk stabilization contributes to stable passive walking in humanoid robots [15], however the dynamics of vestibular organs was not taken into consideration.

In this paper we model the biomechanics of the otolith organ, which is the main source of gravity-related information in the vestibular systems of animals. We use the model to elucidate the benefits of the ubiquitous head stabilization strategy. The understanding of how verticality estimation and head stabilization are achieved in biological systems may lead to better design and control of robotic systems, such as humanlike and animal-like walking robots, or free-roaming drones. The problem of modeling verticality estimation by a nonlinear Newton-method-based observer as well as an extended Kalman filter was previously addressed in [16, 17]. Here, we show empirically that the platform-inertial-measurement system - a strongly nonlinear system that we model in Section II and III-in closed-loop with a linear controller-observer pair yields local linearization of an otherwise fully nonlinear observer-based closed-loop system. Convergence follows from the robustness margin of the full linearization which is demonstrated in Section IV.

\section{Modeling Verticality Estimation}

\section{A. Brief recall of fictitious forces in non-inertial frames: the case of a free or constrained point mass}

From Galileo, we know that the measurement of the gravitational acceleration, $\boldsymbol{g}$, is invariant in a frame moving at constant velocity and which does not rotate. It is the very definition of an 'inertial frame' where all free motions can be explained by a uniform acceleration field. A point located by a vector $\boldsymbol{r}$ in an inertial frame is located in another frame, 1, by ${ }^{1} \boldsymbol{r}=\boldsymbol{r}-\boldsymbol{r}_{1}$, where $\boldsymbol{r}_{1}$ is the vector locating the origin of frame 1 . Let this frame accelerate at rate $\boldsymbol{a}_{1}$, from vector addition, the acceleration of free-falling bodies relatively to 1 is ${ }^{1} \boldsymbol{g}=\boldsymbol{g}-\boldsymbol{a}_{1}$. To an observer fixed with respect to 1 , the quantity $m \boldsymbol{a}_{1}$ is a 'fictitious force' acting on a mass $m$. The tests, $\left|{ }^{1} \boldsymbol{g}\right| \neq|\boldsymbol{g}|$ and ${ }^{1} \dot{\boldsymbol{g}} \neq 0$, can tell an observer that the frame of observation is not inertial. If the frame rotates at rate $\boldsymbol{\omega}_{1}$, there are additional fictitious forces,

$$
{ }^{1} \boldsymbol{g}=\boldsymbol{g}-\underbrace{\dot{\boldsymbol{\omega}}_{1} \times \boldsymbol{r}_{1}}_{\text {Euler }}-\underbrace{2 \boldsymbol{\omega}_{1} \times \boldsymbol{v}_{1}}_{\text {Coriolis }}-\underbrace{\boldsymbol{\omega}_{1} \times\left(\boldsymbol{\omega}_{1} \times \boldsymbol{r}_{1}\right)}_{\text {centrifugal }}-\boldsymbol{a}_{1},
$$

where the Euler term results from the angular acceleration of the moving frame, the Coriolis term forces the mass to remain in a fixed plane of rotation, the centrifugal term tends to force the mass to travel in straight line, and where the last term is due to relative acceleration. An observer moving with frame 1 will see masses fall according to field (1), but for an observer in the inertial frame, the same masses will fall according to $g$. If a mass attached to an ideal force sensor is forced to move, all these terms appear in the measurement since the force $-m^{1} \boldsymbol{g}$ acts on the mass to keep it fixed with respect to the moving frame.

\section{B. Head and otoliths dynamics}

In the vestibular system, the movements of the otolith organs, small solid masses immersed in a viscous fluid, is the response to the gravitational field and to fictitious forces induced by the movements of the head. In an accelerometer, a small test mass is constrained to move along one single direction but responds to the same forces. The otolith masses are constrained to move in two directions. These cases differ only by the constraints imposed on the movements of the test mass. Verticality sensors, such as the otolith organs or inclinometers, can be modeled as damped spherical pendula. Such a pendulum system has two interesting properties. It can be implemented in the form of a liquid-based inclinometer, a device that is free from injurious properties such as hysteresis. The spherical pendulum is also a reasonable model for the otolith organs which gives our results biological relevance. The mechanical model of a platform representing a head and a pendulum representing otolith organs is shown in Fig. 1.
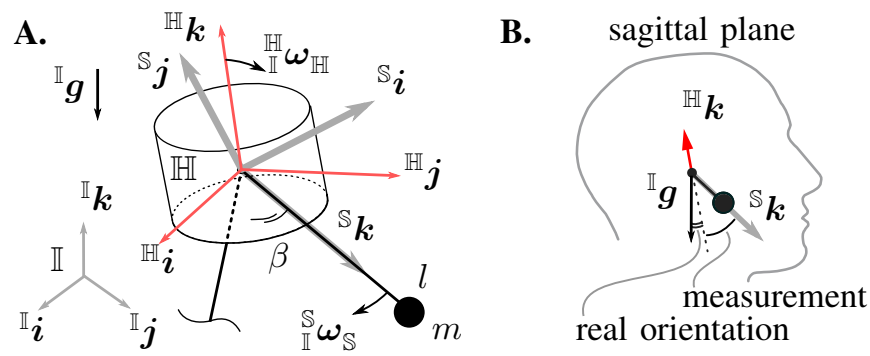

Fig. 1. A. Mechanical system used to model the head and the vestibular system. B. Sagittal plane projection of the system.

Quantities that are sensitive to the frame in which they are expressed are given a left superscript to indicate it. Frame $\mathbb{I}$ is the inertial frame with unit vectors, $\left\{{ }^{\mathbb{I}} \boldsymbol{i},{ }^{\mathbb{I}} \boldsymbol{j},{ }^{\mathbb{I}} \boldsymbol{k}\right\}$; frame $\mathbb{H}$, $\left\{{ }^{\mathbb{H}} \boldsymbol{i},{ }^{\mathbb{H}} \boldsymbol{j},{ }^{\mathbb{H}} \boldsymbol{k}\right\}$, is the body-fixed head-centered frame; frame $\mathbb{S}$, $\left\{{ }^{\mathbb{S}} \boldsymbol{i},{ }^{\mathbb{}} \boldsymbol{j},{ }^{\mathbb{S}} \boldsymbol{k}\right\}$, is the body-fixed pendulum (sensor) coordinate frame, such that its ${ }^{\mathbb{S}} \boldsymbol{k}$ axis is aligned with the arm of the pendulum and the pivot coincides with the center of mass of the head. We model the head, $\mathrm{H}$, as a symmetric rigid body rotating about its center of mass with inertia tensor ${ }^{\mathbb{H}} \mathbf{J}_{\mathrm{H}}=J_{\mathrm{H}} \mathbf{I}_{3}$. The orientation of the head frame, $\mathbb{H}$ w.r.t. $\mathbb{I}$, is described by a rotation matrix, ${ }_{\mathbb{I}} \mathbf{R}_{\mathbb{H}} \in S O(3)$, that transforms by left multiplication vectors expressed in $\mathbb{H}$ to vectors expressed in $\mathbb{I}$. The center of mass of the head coincides with its center of rotation and it is constrained by a spherical joint. The expressions describing the dynamics of multi-body systems are considerably simplified if they are expressed in body-fixed coordinates. For the head, neglecting the influence of the movements of the pendulum, if $\mathbb{\mathbb { H }} \boldsymbol{\omega}_{\mathbb{H}} \in \mathcal{R}^{3}$ expresses the angular velocity of $\mathbb{H}$ w.r.t. $\mathbb{I}$, the dynamics 
forced by a torque ${ }^{\mathbb{H}} \boldsymbol{\tau} \in \mathcal{R}^{3}$ obeys,

$$
\begin{aligned}
{ }^{\mathbb{H}} \mathbf{J}_{\mathrm{H}} \underset{\mathbb{I}}{\mathbb{H}} \dot{\boldsymbol{\omega}}_{\mathbb{H}} & =-{ }_{\mathbb{I}}^{\mathbb{H}} \boldsymbol{\omega}_{\mathbb{H}} \times{ }^{\mathbb{H}} \mathbf{J}_{\mathrm{H}} \mathbb{I} \boldsymbol{\omega}_{\mathbb{H}}+{ }^{\mathbb{H}} \boldsymbol{\tau}, \\
{ }_{\mathbb{I}} \dot{\mathbf{R}}_{\mathbb{H}} & ={ }_{\mathbb{I}} \mathbf{R}_{\mathbb{H}} \mathbb{I} \mathbb{\underline { \omega }} \tilde{\boldsymbol{\omega}}_{\mathbb{H}},
\end{aligned}
$$

where ${ }_{\mathbb{I}}^{\mathbb{H}} \tilde{\boldsymbol{\omega}}_{\mathbb{H}}$ is a skew-symmetric matrix of the platform angular velocity. The orientation of the pendulum frame, $\mathbb{S}$, w.r.t. $\mathbb{I}$, is described by a rotation matrix, ${ }_{\mathbb{I}} \mathbf{R}_{\mathbb{S}}$. The pendulum has a concentrated mass, $m$, a length, $l$, and damping, $\beta$. Let ${ }^{\mathbb{S}} \mathbf{J}_{\mathrm{S}}$ be the inertia tensor of the pendulum, ${ }_{\mathbb{I}}^{\mathbb{S}} \boldsymbol{\omega}_{\mathbb{S}}$ its angular velocity w.r.t. the inertial frame, and ${ }^{\mathbb{S}} \boldsymbol{l}=\left(\begin{array}{lll}0 & 0 & l\end{array}\right)^{\mathrm{T}}$ the vector from the pivot of the pendulum to its center of mass. Noting that ${ }_{\mathbb{S}} \mathbf{R}_{\mathbb{I}}={ }_{\mathbb{I}} \mathbf{R}_{\mathbb{S}}^{\mathrm{T}}$, that ${ }^{\mathbb{I}} \boldsymbol{g}=\left(\begin{array}{lll}0 & 0 & g\end{array}\right)^{\mathrm{T}}$, the equations of motion are found similarly by differentiating the pendulum angular momentum expressed in pendulum coordinates and by adding the terms corresponding to viscous forces and the relative acceleration of the head, ${ }^{\mathbb{H}} \boldsymbol{a} \in \mathcal{R}^{3}$,

$$
\begin{aligned}
{ }^{\mathbb{S}} \mathbf{J}_{\mathrm{S}}{ }_{\mathbb{I}} \dot{\boldsymbol{\omega}}_{\mathbb{S}}= & -{ }_{\mathbb{I}}^{\mathbb{S}} \boldsymbol{\omega}_{\mathbb{S}} \times{ }^{\mathbb{S}} \mathbf{J}_{\mathrm{S}}{ }_{\mathbb{I}} \boldsymbol{\omega}_{\mathbb{S}}+m{ }^{\mathbb{S}} \boldsymbol{l} \times{ }_{\mathbb{S}} \mathbf{R}_{\mathbb{I}}{ }^{\mathbb{I}} \boldsymbol{g} \\
& -\beta\left({ }_{\mathbb{I}}^{\mathbb{S}} \boldsymbol{\omega}_{\mathbb{S}}-{ }_{\mathbb{S}} \mathbf{R}_{\mathbb{I}} \mathbb{I} \boldsymbol{\omega}_{\mathbb{H}}\right)-m{ }^{\mathbb{S}} \boldsymbol{l} \times{ }_{\mathbb{S}} \mathbf{R}_{\mathbb{I} \mathbb{I}} \mathbf{R}_{\mathbb{H}} \mathbb{H} \boldsymbol{a}, \\
{ }_{\mathbb{I}} \dot{\mathbf{R}}_{\mathbb{S}}= & { }_{\mathbb{I}} \mathbf{R}_{\mathbb{S}}{ }_{\mathbb{I}} \tilde{\boldsymbol{\omega}}_{\mathbb{S}},
\end{aligned}
$$

Neglecting the influence the gyroscopic terms, owing to the fact that these terms become negligibly small for low angular velocities and small size devices, the overall model of the head-vestibular-system, despite simplifications, becomes a rather complicated system of forced, nonlinear, coupled differential equations,

$$
\left\{\begin{aligned}
&{ }_{\mathbb{I}} \dot{\boldsymbol{\omega}}_{\mathbb{S}}={ }^{\mathbb{S}} \mathbf{J}_{\mathrm{S}}^{-1}\left[m{ }^{\mathbb{S}} \boldsymbol{l} \times{ }_{\mathbb{S}} \mathbf{R}_{\mathbb{I}}{ }^{\mathbb{I}} \boldsymbol{g}-\beta\left({ }_{\mathbb{I}}^{\mathbb{S}} \boldsymbol{\omega}_{\mathbb{S}}-{ }_{\mathbb{S}} \mathbf{R}_{\mathbb{I}} \mathbb{I} \boldsymbol{\omega}_{\mathbb{H}}\right)\right. \\
&\left.-m{ }^{\mathbb{S}} \boldsymbol{l} \times{ }_{\mathbb{S}} \mathbf{R}_{\mathbb{I} \mathbb{I}} \mathbf{R}_{\mathbb{H}} \mathbb{H} \boldsymbol{a}\right], \\
&{ }_{\mathbb{I}} \dot{\mathbf{R}}_{\mathbb{S}}={ }_{\mathbb{I}} \mathbf{R}_{\mathbb{S}}{ }_{\mathbb{I}} \tilde{\boldsymbol{\omega}}_{\mathbb{S}} \\
& \mathbb{H} \dot{\boldsymbol{\omega}}_{\mathbb{H}}={ }_{\mathbb{H}} \mathbf{J}_{\mathrm{H}}^{-1}{ }_{\mathbb{H}}^{\mathbb{\tau}} \boldsymbol{\tau}, \\
&{ }_{\mathbb{I}} \dot{\mathbf{R}}_{\mathbb{H}}={ }_{\mathbb{I}} \mathbf{R}_{\mathbb{H} \mathbb{I}} \mathbb{H} \tilde{\boldsymbol{\omega}}_{\mathbb{H}} \cdot
\end{aligned}\right.
$$

In biological vestibular systems, it has been observed that the otolith organs in each inner ear respond to the head tilt in frontal and lateral planes. In our model, the angles between the pendulum's body-fixed frame, $\mathbb{S}$, and platform's body fixed frame, $\mathbb{H}$, define the orientation of the head w.r.t. the vertical. In the above model, the orientations are described by rotation matrices whose elements are the projections of unit axes of one coordinate frame into another coordinate frame. Then, the following expressions can be used to express the angles between the pendulum and head frames in the frontal and lateral planes through the projections of the unit vectors,

$$
\tan \phi_{x}=\frac{\left[\mathbb{H} \mathbf{R}_{\mathbb{S}}\right]_{\{3,2\}}}{\left[{ }_{\mathbb{H}} \mathbf{R}_{\mathbb{S}}\right]_{\{3,3\}}}, \quad \tan \phi_{y}=\frac{\left[{ }_{\mathbb{H}} \mathbf{R}_{\mathbb{S}}\right]_{\{3,1\}}}{\left[{ }_{\mathbb{H}} \mathbf{R}_{\mathbb{S}}\right]_{\{3,3\}}},
$$

where ${ }_{\mathbb{H}} \mathbf{R}_{\mathbb{S}}={ }_{\mathbb{I}} \mathbf{R}_{\mathbb{H}}^{\mathrm{T}} \mathbf{R}_{\mathbb{S}}$ and the notation $[\mathbf{M}]_{\{n, m\}}$ represents the element of the matrix $\mathbf{M}$ in the $n$-th row and $m$-th column. Angles $\phi_{x}, \phi_{y}$ are the angles between the projections of ${ }^{\mathbb{S}} \boldsymbol{k}$ to the frontal and lateral head planes and ${ }^{\mathbb{H}} \boldsymbol{k}$, respectively. Eqs. (3) express the measurements (outputs) for the system (2) which can model the otolith responses in the human vestibular system.

\section{Modeling Head Stabilization}

We define that the head is horizontally stabilized (or upright) when the heads longitudinal axis is aligned with the gravitational vertical. Pursuing the goal of stabilizing the head around the vertical from idiothetic measurements requires the design of an observer to extract the true vertical from the sensor movements known in head coordinates. The results are fed to a controller, which naturally calls for the application of the separation principle. Unfortunately, the application this principle to nonlinear systems is still an active topic of theoretical research with sparse results that apply to restricted classes of systems [18, 19]. In the following, the separation principle is considered and the controller and observer designs is carried out for a system linearized around an equilibrium.

Control design. A possible danger when using simplified control and observation can be the small region of convergence for the controller and for the estimator. However, global attitude control laws for rigid bodies in $S O(3)$ are known,[20]

$$
{ }^{\mathbb{H}} \boldsymbol{\tau}=-\boldsymbol{K}_{\mathrm{p}} \boldsymbol{\Omega}-\boldsymbol{K}_{\mathrm{d}} \mathbb{\mathbb { H }} \boldsymbol{\omega}_{\mathbb{H}}, \text { with } \boldsymbol{\Omega} \equiv \sum_{i=1}^{3} e_{i} \times\left(\mathbf{R}_{\mathbb{I}}^{\mathrm{d}} \mathbf{R}_{\mathbb{H}} e_{i}\right),
$$

where $\boldsymbol{K}_{\mathrm{p}}=k_{\mathrm{p}} \mathbf{I}_{3}$ and $\boldsymbol{K}_{\mathrm{d}}=k_{d} \mathbf{I}_{3}$, are symmetric, positive definite control gain matrices, $\left(\begin{array}{lll}e_{1} & e_{2} & e_{3}\end{array}\right)=\mathbf{I}_{3}$, and $\mathbf{R}^{\mathrm{d}}$ is the desired rotation matrix. When the desired rotation is the identity matrix, $\mathbf{R}^{\mathrm{d}}=\mathbf{I}_{3}$, this control law stabilizes the head platform horizontally from any initial conditions. An alternate, simpler design may merely employ the head tilt angles in the frontal and lateral planes and the head angular velocity are the inputs,

$$
\left\{\begin{array}{l}
{ }^{\mathbb{H}} \boldsymbol{\tau}_{x}=k_{\mathrm{p}, x}\left(\phi_{x}^{\mathrm{d}}-\arctan \frac{\left[_{\mathbb{I}} \mathbf{R}_{\mathbb{H}}\right]_{\{3,2\}}}{\left.{ }_{\mathbb{I}} \mathbf{R}_{\mathbb{H}}\right]_{\{3,3\}}}\right)-k_{\mathrm{d}, x} \mathbb{I} \mathbb{I} \boldsymbol{\omega}_{\mathbb{H} x}, \\
{ }^{\mathbb{H}} \boldsymbol{\tau}_{y}=k_{\mathrm{p}, y}\left(\phi_{y}^{\mathrm{d}}-\arctan \frac{\left[{ }_{\mathbb{I}} \mathbf{R}_{\mathbb{H}}\right]_{\{3,1\}}}{\left[{ }_{\mathbb{I}} \mathbf{R}_{\mathbb{H}}\right]_{\{3,3\}}}\right)-k_{\mathrm{d}, y} \mathbb{\mathbb { H }} \boldsymbol{\omega}_{\mathbb{H} y} .
\end{array}\right.
$$

where $k_{p}, k_{d}$ are the control gains and $\phi_{x}^{\mathrm{d}}$ and $\phi_{y}^{\mathrm{d}}$ are the desired frontal and lateral tilts of the head w.r.t. the inertial frame. When the platform is close to be horizontal, ${ }_{\mathbb{I}} \mathbf{R}_{\mathbb{H}} \approx \mathbf{I}_{3}$, (4) can be further linearized around the horizontal orientation,

$$
\left\{\begin{array}{c}
{ }^{\mathbb{H}} \boldsymbol{\tau}_{x}=k_{\mathrm{p}, x}\left(\phi_{x}^{\mathrm{d}}-\left[{ }_{\mathbb{I}} \mathbf{R}_{\mathbb{H}}\right]_{\{3,2\}}\right)-k_{\mathrm{d}, x} \mathbb{\mathbb { H }} \boldsymbol{\omega}_{\mathbb{H} x}, \\
{ }^{\mathbb{H}} \boldsymbol{\tau}_{y}=k_{\mathrm{p}, y}\left(\phi_{y}^{\mathrm{d}}-\left[{ }_{\mathbb{I}} \mathbf{R}_{\mathbb{H}}\right]_{\{3,1\}}\right)-k_{\mathrm{d}, y} \mathbb{\mathbb { H }} \boldsymbol{\omega}_{\mathbb{H} y}
\end{array}\right.
$$

The orientation of the head w.r.t. the inertial frame, ${ }_{\mathbb{I}} \mathbf{R}_{\mathbb{H}}$, must be observed.

Observer design. Let $\boldsymbol{x}=\left({ }_{\mathbb{I}}^{\mathbb{S}} \boldsymbol{\omega}_{\mathbb{S}},{ }_{\mathbb{I}} \mathbf{R}_{\mathbb{S}},{ }_{\mathbb{I}}^{\mathbb{H}} \boldsymbol{\omega}_{\mathbb{H}},{ }_{\mathbb{I}} \mathbf{R}_{\mathbb{H}}\right)^{\mathrm{T}}$ be a state vector made of the elements of the vectors and matrices arranged in a single vector. The system (2) and outputs (3) are then conveniently expressed in the form,

$$
\dot{\boldsymbol{x}}=f(\boldsymbol{x}, \boldsymbol{u}), \quad \boldsymbol{y}=h(\boldsymbol{x}),
$$

where $\boldsymbol{u}=\left({ }^{\mathbb{H}} \boldsymbol{a},{ }^{\mathbb{H}} \boldsymbol{\tau}\right)^{\top}$ is an input due to the translational movement of the head combined with the torque applied to it. Linearizing the system around the horizontal orientation 
A.
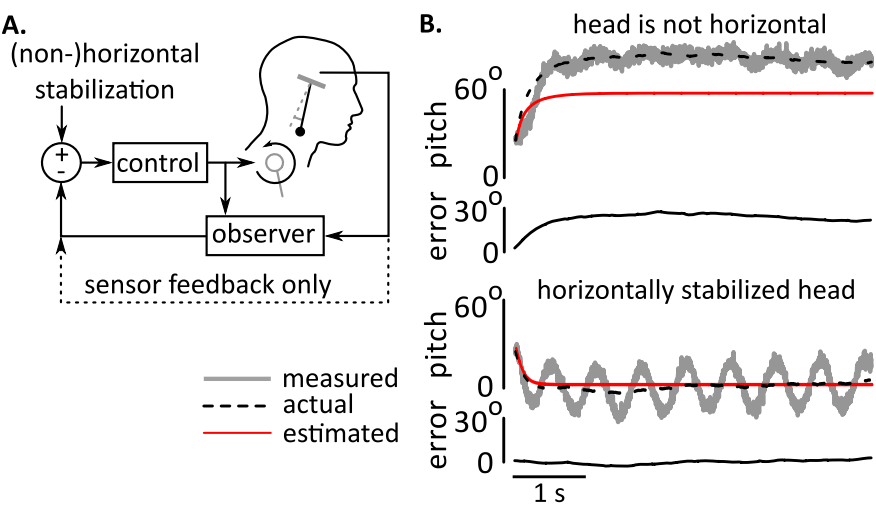

Fig. 2. A, Control diagram for simulation and experimental validations. B, Simulation results for Kalman filter for the cases of non-horizontal and horizontal head stabilization.
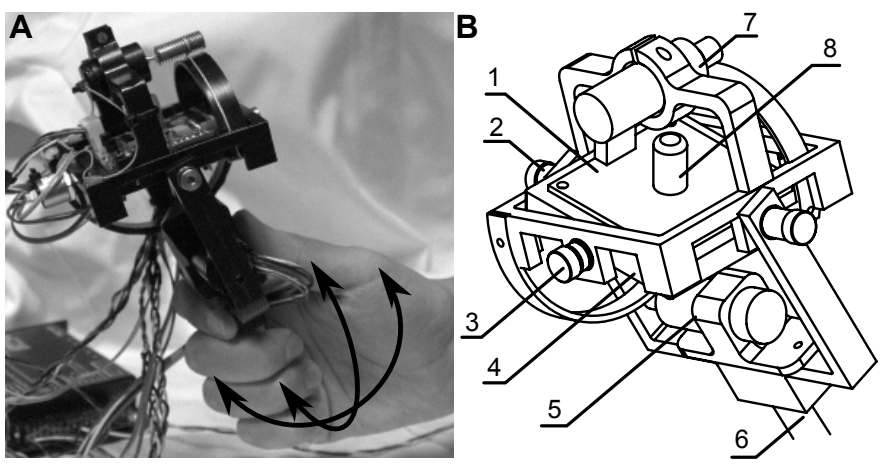

Fig. 3. Experimental setup. A, Platform stabilization experiment. The base link of the gimbal mechanism was moved manually. The task of the controller was to stabilize the platform horizontally. B, Mechanical design. 1 - gimbal's platform with inclinometer; 2 - rotational joint with encoder (frontal tilt); 3 - rotational joint with encoder (lateral tilt); 4 - MEMS accelerometer; 5 actuator for frontal tilt; 6 - base link; 7 - actuator for lateral tilt; 8 - liquid based inclinometer.

$\boldsymbol{x}_{0}=[(0,0,0), \mathbf{I},(0,0,0), \mathbf{I}]^{\mathrm{T}}$, where the platform's rotation matrix is identity and the angular velocity zero,

$$
\dot{\boldsymbol{x}}=\mathbf{A} \boldsymbol{x}+\mathbf{B} \boldsymbol{u}, \boldsymbol{y}=\mathbf{C}(\boldsymbol{x}),
$$

with constant matrices,

$$
\mathbf{A}=\left.\frac{\partial f}{\partial \boldsymbol{x}}\right|_{\substack{\boldsymbol{x}=\boldsymbol{x}_{0} \\ \boldsymbol{a}=0}}, \mathbf{B}=\left.\frac{\partial f}{\partial \boldsymbol{u}}\right|_{\substack{\boldsymbol{x}=\boldsymbol{x}_{0} \\ \boldsymbol{a}=0}}, \mathbf{C}=\left.\frac{\partial h}{\partial \boldsymbol{x}}\right|_{\substack{\mathbb{H}=\boldsymbol{x}_{0} \\ \boldsymbol{a}=0}}
$$

A linear state observer (Luenberger observer [21]) for the linearized system is of the following form:

$$
\dot{\hat{\boldsymbol{x}}}=\mathbf{A} \hat{\boldsymbol{x}}+\mathbf{B} \boldsymbol{u}+\mathbf{L}(\boldsymbol{y}-\mathbf{C} \hat{\boldsymbol{x}}),
$$

where $\hat{\boldsymbol{x}}$ is the estimated state and $\mathbf{L}$ the observer gain matrix. We could employ a Kalman filter to estimate the state when the measurements are noisy. The observer gain, $\mathbf{L}$, in (7) is then replaced by a Kalman gain and standard design equations can be used.

\section{REsults}

Simulation. The parameters of the verticality sensor were those of the model of type later in the experiments (Model 900,
Applied Geomechanics). For a critically damped sensor with a natural frequency of $10 \mathrm{~Hz}$ we get the following parameter values: $\beta /\left(m^{\mathbb{S}} \boldsymbol{l}^{2}\right)=126 \mathrm{~N} \cdot \mathrm{s} / \mathrm{kg} \cdot \mathrm{m}^{2}$ and $g /{ }^{\mathbb{S}} \boldsymbol{l}=39441 / \mathrm{s}^{2}$ with $g=9.81 \mathrm{~m} / \mathrm{s}^{2}$. The head inertia was taken to be $J_{\mathrm{H}}=0.0001 \mathrm{~kg} \cdot \mathrm{m}^{2}$. For simplicity, we present results for only one tilt value of the platform. In the simulation scenarios, the platform was accelerated at an oscillatory rate, ${ }^{\mathbb{H}} \boldsymbol{a}=[0,3 \sin (10 t), 0]^{\mathrm{T}}$. A PD-regulator with control gains $k_{\mathrm{p}}=1 \mathrm{~N} / \mathrm{m} / \mathrm{rad}$ and $k_{\mathrm{d}}=0.1 \mathrm{~N} \cdot \mathrm{s} / \mathrm{m} / \mathrm{rad}$ was used. The platform initial orientation was set to $23^{\circ}$. The numerical integration of the system equations was performed with a $1 \mathrm{~ms}$ time-step. Fig. 2A shows a simplified diagram of the observer-based control system configuration which was used in simulations and experimental validation. Depending on the test scenario the head angular orientation was controlled based on direct sensor feedback or else based on estimated state feedback.

The head was accelerated while the controller tried to maintain the orientation of the head at $60^{\circ}$ in the first case, Fig. 2B, and at $0^{\circ}$ in the second case, Fig. 2D. The estimated head's pitch and angular velocity were fed back to the PDregulator. As the plots show, the actual tilt was greater than $60^{\circ}$ which meant that the PD-regulator was receiving incorrect estimates of the state. This happened because of the approximation introduced by the linearization of the model around the horizontal orientation for the observer design. The estimation error was reduced from $\approx 30^{\circ}$ to $\approx 4^{\circ}$ when the platform was horizontally stabilized. When noise was introduced to the measurements (standard deviation of process noise $0.06^{\circ}$ and measurement noise $1^{\circ}$ ), tests with a Kalman filter showed similar performance. Moreover, uncertainty in model parameters values up to $10 \%$ did not affect performance.

Experiment. To test the proposed verticality estimation by the head stabilization strategy, we constructed a two degreesof-freedom (2-dof) gimbal mechanism which could be held in the hand as shown in Fig. 3A. Two motors (Maxon Model ECpowermax $22 \mathrm{~mm}$, Faulhaber Model $024 \mathrm{SR}$ ) controlled the angular orientation of the platform. The mechanical details, Fig. 3B, show how the two motors acted to the axes of the gimbal via two capstan drives. The liquid-based inclinometer (model T900, Applied Geomechanics) provided two decoupled orientation measurements based on the degree of immersion of four electrodes in a liquid contained in a vial. The platform formed the distal link of the gimbal mechanism. The inclinometer was located near the center of rotation. The observation and control algorithms ran on a Gumstix computer with a sampling period of $5 \mathrm{~ms}$. A MEMs-based 3-axis accelerometer (ST Microelectronics, Model LIS344ALH) was mounted on the platform in the vicinity of the inclinometer. Two potentiometers (US Digital Model MA3) were mounted on the gimbal axes for joint angle monitoring. They were not used for control.

The system dynamics were identified. The two drives were modeled as first order mass-damper system with an integrator. Pitch and roll rotations were identified independently from the response to step inputs. The orientation of the platform 

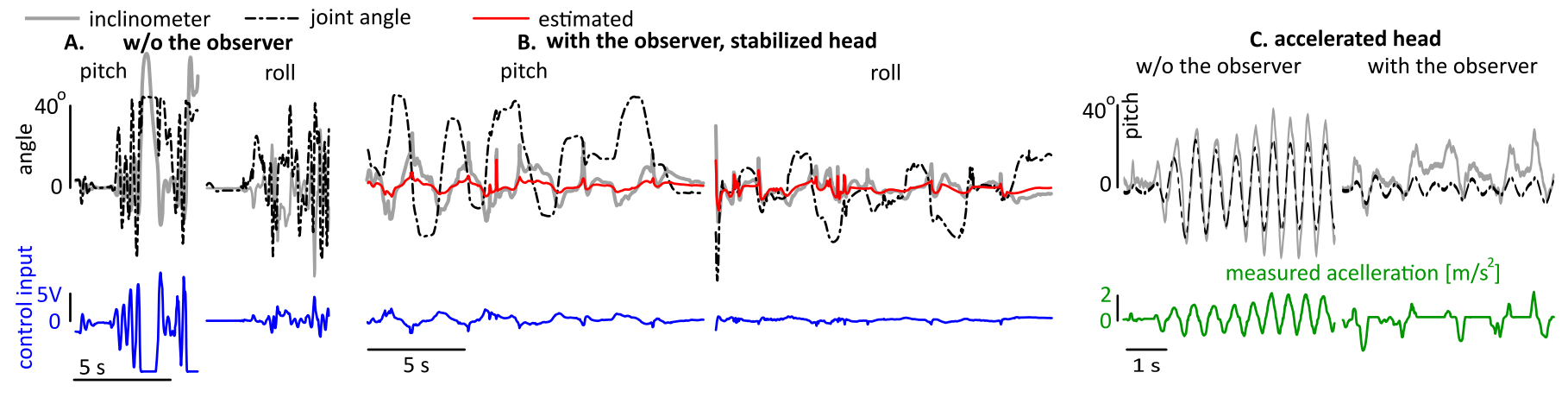

Fig. 4. Experimental results. A: stabilization based on direct roll and pitch measurements from the inclinometer. B: stabilization based on the estimated head's orientation. The system was stable. C: head stabilization during horizontal acceleration based on direct inclinometer measurements (left panel) and based on state estimation (right panel).

was measured as an output. The dynamic model parameters were identified with the help of Matlab Identification Toolbox. For the pitch, the parameter values were: moment of inertia $J_{1}=0.008 \mathrm{~kg} \cdot \mathrm{m}^{2}$, damping $b_{1}=1.35 \mathrm{~N} \cdot \mathrm{m} \cdot \mathrm{s}$. For the roll, the parameters were: moment of inertia $J_{2}=0.0006 \mathrm{~kg} \cdot \mathrm{m}^{2}$, damping $b_{2}=0.137 \mathrm{~N} \cdot \mathrm{m} \cdot \mathrm{s}$. A second order system was used to model the inclinometer dynamics. During the experiments, the observer received measurements from the inclinometer and from the accelerometer.

Experimental results. We experimentally tested two cases: sensor-based direct feedback and observer-based feedback. The task of the controller was to stabilize the platform horizontally in spite of any random motions of the mechanism base link. The potentiometers monitored the angular orientation of the head with respect to the base link. When the head was horizontally stabilized their measurements provided the orientation of the base link with respect to the estimated gravitational vertical.

We first tested the case when stabilization was achieved by direct feedback of the pitch and roll measurements were fed to the PD-regulator. The base link was oscillated at a magnitude of $40-50^{\circ}$. Fig. $4 \mathrm{~A}$ shows the results. The first row displays the time history of the inclinometer measurements as well as the joint angular measurements of the platform with respect to the base link. The inclinometer measurements lagged behind the angular measurements since the liquid in the capsule had slow dynamics. The platform became unstable. The second row of Fig. 4A shows the control input given by the PD-controllers.

In the observer-based stabilization test, the base link was again moved with $40-50^{\circ}$ oscillations. Fig. 4B shows the estimated angular orientation of the platform. The tilt measurements from the inclinometer reached $20^{\circ}$ and the system remained stable. The first row displays the time history of the inclinometer measurements, the platform angular measurements, and the estimated platform absolute pitch and roll angles. The second row shows the control inputs. The magnitude of the platform oscillations in absolute coordinates were significantly smaller than the magnitudes of the relative angular oscillations.

The system was then accelerated back-and-forth with an acceleration of about $2 \mathrm{~m} / \mathrm{s}^{2}$. The results are shown in Fig. $4 \mathrm{C}$, left panel. The first row shows the relative head orientation. The second row shows the acceleration of the head. The system quickly became unstable due to disturbed inclinometer measurements with large spontaneous oscillations. The accelerometer attached to the platform was strongly influenced by the movements and was not providing accurate information.

Fig. 4C, right panel, shows the results when the observer was used for head stabilization during acceleration. The head was linearly accelerated while the PD-regulator forced the platform to remain horizontal. The platform oscillated with a magnitude smaller than $6^{\circ}$, which was much smaller than in the first experiment. The accelerometer measurements were much less influenced.

\section{CONClusion}

We performed simulations and experiments to study the head stabilization strategy that is universally observed in nature with a view to apply it in robotics. It was shown that this strategy could provide gravitational verticality estimates based on pure inertial measurements in a non-inertial frame. When an inertial platform is maintained horizontal by observation and feedback, the measurements respond to translational movements only and angular components are rejected. It therefore becomes straightforward to estimate the unknown linear acceleration by solving the dynamics equations of the sensing device. This result hinges on the applicability of the separation principle when the controller and the observer are designed independently. We surmise that this principle could apply because the head stabilization strategy linearized the closed loop system sufficiently.

In terms of application to robotics, the stabilization was effective even with simplified linear control, and results in dramatically improved verticality estimation. Locating IMU in a robot's head and implementing the head stabilization strategy has been recently demonstrated in several humanoid robots applications with a view to mimic human gaze stabilization behavior [22]; to improve gaze stabilization for visual tracking [23], and to efficiently coordinate the robot's body movement [23, 24]. Whereas some progress has been achieved in transferring anthropomorphic behaviors to humanoid robots, 
substantial research and development challenges remain in particular the role of head stabilization in resolution of gravitoinertial ambiguity. Resolution of gravito-inertial ambiguity for inertial sensor measurements is problematic for robots with idiothetic sensing and very often employment of external sensors with a fixed frame of reference is required. The present work addressed this issue through modeling the sensor's and the head's dynamics with closed loop head stabilization controller and observer. The result presented here complements recent developments in the field [22, 23, 24, 25] by demonstrating that head stabilization significantly improves the quality of verticality estimation. This result has many applications in the control of humanoid robots, rovers, drones, as well as human mobility assistance robots [26, 27].

Gravity-based inertial measurements are the only absolute sources of information about the gravitational verticality. We believe that inertially stabilized platforms could provide for the development of ground-independent locomotion strategies in future robots. Such robots would be free of the assumption of interacting with firm, flat, and horizontal grounds. Posture control could then be realized in top-to-down manner, from a stabilized platform down to the robot appendages.

\section{REFERENCES}

[1] D. E. Angelaki and K. E. Cullen, "Vestibular system: the many facets of a multimodal sense," Annu. Rev. Neurosci., vol. 31, pp. 125-150, 2008.

[2] A. R. Bisdorff, C. J. Wolsley, D. Anastasopoulos, A. M. Bronstein, and M. A. Gresty, "The perception of body verticality (subjective postural vertical) in peripheral and central vestibular disorders," Brain, vol. 119, no. 5, pp. 1523-1534, 1996.

[3] I. Farkhatdinov, H. Michalska, A. Berthoz, and V. Hayward, Review of anthropomorphic head stabilisation and verticality estimation in robots. Springer, 2019, pp. 185-209.

[4] T. Pozzo, A. Berthoz, and L. Lefort, "Head stabilisation during various locomotor tasks in humans," Exp Brain Res, vol. 82, no. 1, pp. 97-106, 1990.

[5] T. Pozzo, Y. Levik, and A. Berthoz, "Head and trunk movements in the frontal plane during complex dynamic equilibrium tasks in humans," Exp Brain Res, vol. 106, no. 2, pp. 327-338, 1995.

[6] D. Guitton, R. E. Kearney, N. Wereley, and B. W. Peterson, "Visual, vestibular and voluntary contributions to human head stabilization," Exp Brain Res, vol. 64, no. 1, 1986.

[7] A. M. Bronstein, "Evidence for a vestibular input contributing to dynamic head stabilization in man," Acta Oto-Laryngologica, vol. 105, no. 1-2, pp. 1-6, 1998.

[8] R. Grasso, P. Prévost, Y. P. Ivanenko, and A. Berthoz, "Eyehead coordination for the steering of locomotion in humans: an anticipatory synergy," Neurosci. Lett, vol. 253, no. 2, pp. 115-118, 1998.

[9] R. A. Brooks, "The cog project," Journal of the Robotics Society of Japan, vol. 15, no. 7, pp. 968-970, 1997.

[10] A. Paramiggiani, M. Maggiali, L. Natale, F. Nori, A. Schmitz, N. Tsagarakis, J. S. Victor, F. Becchi, G. Sandini, and G. Metta, "The design of the icub humanoid robot," Int J of Humanoid Robotics, 2012.

[11] T. Asfour, P. Azad, N. Vahrenkamp, K. Regenstein, A. Bierbaum, K. Welke, J. Schröder, and R. Dillmann, "Toward humanoid manipulation in human-centred environments," Robot Auton Syst, vol. 56, no. 1, pp. 54-65, 2008.

[12] S.-H. Hyon, J. G. Hale, and G. Cheng, "Full-body compliant human-humanoid interaction: balancing in the presence of unknown external forces," IEEE T on Robotics, vol. 23, no. 5, pp. 884-898, 2007.

[13] P. Kryczka, E. Falotico, K. Hashimoto, H. Lim, A. Takanishi, C. Laschi, P. Dario, and A. Berthoz, "Implementation of a human model for head stabilization on a humanoid platform," in IEEE RAS\&EMBS Int Conf Biomed Robotics and Biomechatronics, 2012, pp. 675-680.

[14] E. Falotico, N. Cauli, K. Hashimoto, P. Kryczka, A. Takanishi, P. Dario, A. Berthoz, and C. Laschi, "Head stabilization based on a feedback error learning in a humanoid robot," in IEEE ROMAN, 2012, pp. 449-454.

[15] M. Benallegue, J.-P. Laumond, and A. Berthoz, "Contribution of actuated head and trunk to passive walkers stabilization," in IEEE Int Conf on Robotics and Automation,, May 2013, pp. $5638-5643$.

[16] I. Farkhatdinov, V. Hayward, and A. Berthoz, "On the benefits of head stabilization with a view to control balance and locomotion in humanoids," in IEEE Int Conf on Humanoid Robots, 2011, pp. 147-152.

[17] I. Farkhatdinov, H. Michalska, A. Berthoz, and V. Hayward, "Modeling verticality estimation during locomotion," in Romansy 19-Robot Design, Dynamics and Control. Springer, 2013, pp. 359-366.

[18] A. Golubev, A. Krishchenko, and S. Tkachev, "Separation principle for a class of nonlinear systems," in Proc 15-th IFAC World Congress, 2002.

[19] C.-W. Park and S. Lee, "Local separation principle for a special class of nonlinear systems," International Mathematical Forum, vol. 1, no. 40, pp. 1983-1995, 2007.

[20] N. Chaturvedi, A. Sanyal, and N. McClamroch, "Rigid-body attitude control," IEEE Control Systems, vol. 31, no. 3, pp. 3051, 2011.

[21] D. G. Luenberger, "Observing the state of a linear system," IEEE T. Mil. Electron., vol. 8, no. 2, pp. 74-80, 1964.

[22] N. Pateromichelakis, A. Mazel, M. Hache, T. Koumpogiannis, R. Gelin, B. Maisonnier, and A. Berthoz, "Head-eyes system and gaze analysis of the humanoid robot romeo," in 2014 IEEE/RSJ Int Conf on Intelligent Robots and Systems, 2014, pp. 1374-1379.

[23] L. Vannucci, S. Tolu, E. Falotico, P. Dario, H. H. Lund, and C. Laschi, "Adaptive gaze stabilization through cerebellar internal models in a humanoid robot," in 2016 6th IEEE Int Conf on Biomedical Robotics and Biomechatronics (BioRob), 2016, pp. 25-30.

[24] E. Falotico, N. Cauli, P. Kryczka, K. Hashimoto, A. Berthoz, A. Takanishi, P. Dario, and C. Laschi, "Head stabilization in a humanoid robot: models and implementations," Autonomous Robots, vol. 41, no. 2, pp. 349-365, 2017.

[25] I. Farkhatdinov, H. Michalska, A. Berthoz, and V. Hayward, "Gravito-inertial ambiguity resolved through head stabilization," Proc. R. Soc. A, vol. 475, no. 20180010, pp. 414-421, 2019.

[26] I. Farkhatdinov, J. Ebert, G. van Oort, M. Vlutters, E. van Asseldonk, and E. Burdet, "Assisting human balance in standing with a robotic exoskeleton," IEEE Robot. Autom. Lett., vol. 4, no. 2, pp. 414-421, 2019.

[27] I. Farkhatdinov, N. Roehri, and E. Burdet, "Anticipatory detection of turning in humans for intuitive control of robotic mobility assistance," Bioinspir Biomim., vol. 12, no. 5, p. 055004, 2017. 